\title{
Development and validation of the brief esophageal dysphagia questionnaire
}

\author{
T. H. TAFT, ${ }^{\star}$ M. RIEHL,$\dagger$ J. B. SODIKOFF, ${ }^{\star}$ P. J. KAHRILAS, ${ }^{\star}$ L. KEEFER, \\ ${ }^{\star}$ Division of Gastroenterology \& Hepatology, Northwestern University Feinberg School of Medicine, Chicago, IL, USA \\ $\dagger$ Division of Gastroenterology, University of Michigan, Ann Arbor, MI, USA \\ $\ddagger$ Icahn School of Medicine, Mount Sinai Medical Center, Susan and Leonard Feinstein IBD Center, New York, NY, USA
}

\section{Key Points}

- Esophageal dysphagia is a commonly occurring symptom in clinical practice. Existing measures are either lengthy and, thus, impractical for clinical practice or do not evaluate the presence of food impaction.

- The Brief Esophageal Dysphagia Questionnaire (BEDQ) is a reliable and valid rapid assessment tool for esophageal dysphagia that also identifies food impaction symptoms.

- The BEDQ is easily implemented in clinical practice and may serve as a guide for further clinical evaluation of patients presenting with esophageal dysphagia.

\begin{abstract}
Background Esophageal dysphagia is common in gastroenterology practice and has multiple etiologies. A complication for some patients with dysphagia is food impaction. A valid and reliable questionnaire to rapidly evaluate esophageal dysphagia and impaction symptoms can aid the gastroenterologist in gathering information to inform treatment approach and further evaluation, including endoscopy. Methods 1638 patients participated over two study phases. 744 participants completed the Brief Esophageal Dysphagia Questionnaire (BEDQ) for phase 1; 869 completed the BEDQ, Visceral Sensitivity Index, Gastroesophageal Reflux Disease Questionnaire, and Hospital Anxiety and Depression Scale for phase 2. Demographic and clinical data were obtained via the electronic medical record. The BEDQ was evaluated for internal consistency, split-half reliability, ceiling and floor effects, and construct validity. Key Results The BEDQ demonstrated excellent internal consistency, reliability, and construct validity. The
\end{abstract}

Address for Correspondence

Dr. Tiffany Taft, Clinical Research Associate, 676 N. St. Clair

St., Suite 1400, Chicago, IL 60611, USA.

Tel: +1 312725 6175; fax: +1 312695 3999;

e-mail: ttaft@northwestern.edu

Received: 12 March 2016

Accepted for publication: 27 May 2016 symptom frequency and severity scales scored above the standard acceptable cutoffs for reliability while the impaction subscale yielded poor internal consistency and split-half reliability; thus the impaction items were deemed qualifiers only and removed from the total score. No significant ceiling or floor effects were found with the exception of 1 item, and inter-item correlations fell within accepted ranges. Construct validity was supported by moderate yet significant correlations with other measures. The predictive ability of the BEDQ was small but significant. Conclusions et Inferences The BEDQ represents a rapid, reliable, and valid assessment tool for esophageal dysphagia with food impaction for clinical practice that differentiates between patients with major motor dysfunction and mechanical obstruction.

Keywords esophageal dysphagia, food impaction, questionnaire validation.

\section{INTRODUCTION}

Esophageal dysphagia is a common symptom in gastroenterology practices and is loosely defined as 'difficulty with swallowing'. Mechanistically, this type of dysphagia is separated into structural, associated with an overt obstruction, and non-structural, 
associated with no evidence of an anatomical cause on endoscopy., ${ }^{1,2}$ A subset of patients with esophageal dysphagia experience acute food impaction, which has an annual incidence of approximately 13 cases per 100000 people per year and is the third most common non-billiary endoscopic emergency after upper and lower gastrointestinal hemorrhage. ${ }^{3}$ The main causes of food impaction are structural diseases such as esophageal stricture, Schatzki ring, and eosinophilic esophagitis. ${ }^{3-8}$ However, food impaction also occurs in conditions characterized by abnormal motor function (e.g. achalasia) and distinguishing these entities by history alone may be difficult.

Non-structural esophageal dysphagia can present with symptoms related to poor bolus transport and obstruction at the lower esophageal sphincter due to abnormal motor function. ${ }^{9}$ Abnormal perception secondary to visceral hypersensitivity in the context of normal or borderline motor function also leads to dysphagia symptoms. ${ }^{10-12}$ In these cases, esophageal dysphagia is often accompanied by other symptoms including regurgitation, heartburn, and chest pain, ${ }^{13}$ further complicating the assessment of this symptom simply as 'trouble swallowing'.

While several measures have been developed to evaluate oropharyngeal dysphagia ${ }^{14}$ fewer exist to evaluate esophageal dysphagia; these include the Mayo Dysphagia Questionnaire - 30 (MDQ30), ${ }^{15}$ the Esophageal Symptoms Questionnaire (ESQ), ${ }^{16}$ the Dysphagia Symptom Questionnaire (DSQ) ${ }^{17}$ and the Eosinophilic Esophagitis Symptom Activity Index (EEsAI-PRO). ${ }^{18}$ The MDQ30 is a comprehensive assessment tool designed to gauge dysphagia frequency and severity in the past 30 days, and the presence of gastroesophageal reflux disease (GERD) or allergic symptoms, medication use, and prior surgical history for dysphagia; the ESQ is a similar measure that gauges frequency and intensity of esophageal symptoms including dysphagia, reflux, and globus. The DSQ and EEsAI are disease-specific measure for Eosinophilic Esophagitis that could be tailored to other conditions. Due to their length, the EEsAI, MDQ30, and ESQ may be challenging to implement in day-to-day clinical practice. The DSQ is short, being only 3 items, but requires repeated measurement via an electronic diary and thus, is not conducive to an outpatient clinic setting. An additional scale, the Straumann Dysphagia Instrument (SDI), is a brief questionnaire to measure dysphagia severity; however, as of the preparation of this manuscript the SDI is not validated. ${ }^{17,19}$ These limitations to current measures present an important limitation in the management of esophageal dysphagia, as simple reliable measures of symptom severity in the outpatient setting could be utilized to follow patients before and after interventions to inform future treatment options.

We aimed to develop a rapid assessment tool (less than $5 \mathrm{~min}$ ) to identify the presence and severity of esophageal dysphagia symptoms, including experiences with food impaction, to aid clinicians in gathering information during outpatient visits. Our secondary goal was to create a general symptom score that could be utilized in both structural and nonstructural disease processes to simplify the reporting process for both the patient and healthcare provider. We hypothesized that this measure could be utilized to help make decisions regarding the requirement for further diagnostic testing and whether endoscopic or surgical interventions are needed.

\section{MATERIALS AND METHODS}

This study was conducted in two phases: phase 1 data evaluated the internal consistency and reliability (measured via Cronbach alpha and the Guttman statistic for split-half reliability) and the factor structure (measured via Principle Components Factor Analysis) of the BEDQ, phase 2 data evaluated the concurrent, construct, and predictive validity of the measure. For both study phases, potential participants ages 18 and older with a documented ICD-9 diagnosis of GERD or dysphagia NOS (Not Otherwise Specified) for at least 6 months were approached consecutively during their routine visit to a university-based outpatient gastroenterology clinic. Participants in phase 2 also had one high resolution esophageal manometry performed at the study center prior to study enrollment documented in the electronic medical record (EMR). Informed consent was obtained and clinical data outlined below were prospectively collected via the EMR at the time of participant consent. Participants also completed study questionnaires.

\section{Phases 1 and 2}

Demographic information: gender, age, race, ethnicity Clinical data: gastrointestinal diagnosis-Brief Esophageal Dysphagia Questionnaire (Tested version; BEDQ) - Preliminary Version: The initial version of the BEDQ is a 10-item self-report measure of dysphagia symptom frequency (5 items), severity (3 items), and impaction (2 items). Symptom frequency (e.g. 'trouble eating solid food (meat, bread)', 'coughing or choking while swallowing foods or liquids') is measured on a 6-point Likert scale from 'never' to 'daily' during the past 30 days. Symptom severity (e.g. 'Eating solid food [meat, bread, vegetables]', 'drinking liquids') is measured on a 6-point Likert scale from 'none' to 'severe' during the past 180 days. Impactions (e.g. 'an emergency room visit because of food being stuck in throat or esophagus') are measured on a 6point Likert scale from 'never' to 'more than four times' during the past 12 months. Items on the BEDQ were selected by consensus from two gastroenterologists with expertise in treating dysphagia and other esophageal conditions and are scored from 0 to 5. Higher scores indicate greater impaction dysphagia. The BEDQ takes 1-2 min to complete. The final version of the BEDQ, taking into account modifications based on study findings, is found in Appendix S1. 


\section{Phase 2}

Additional clinical data Esophageal manometry results (esophagogastric junction outflow obstruction, esophageal spasm, absent contractility, hypercontractility) categorized patients as having major motor dysfunction (yes/no). Endoscopy and/or esophagram results (stricture, eosinophilic esophagitis, mass, postsurgical conditions, cricopharyngeal bar) categorized patients as having a mechanical obstruction (yes/no), and large $(>5 \mathrm{~cm})$ hiatal hernia (yes/no). Distal contractile integral (DCI) and integrated relaxation pressure (IRP) values provided gold standard assessment points of esophageal dysfunction severity. Body mass index (BMI) was also recorded.

Visceral sensitivity index The visceral sensitivity index (VSI) is a 15 item self-report measure of a patient's gastrointestinal symptom-specific anxiety within five domains: worry, fear, vigilance, sensitivity, and avoidance. ${ }^{20}$ Items are rated on a 6-point Likert scale (strongly agree to strongly disagree) and yield an overall score. The VSI is widely used in gastrointestinal research, and demonstrates excellent reliability (Cronbach $\alpha=0.93$ ) and validity. Higher scores indicate greater visceral anxiety.

Hospital Anxiety $\leftrightarrow$ Depression Scale The Hospital Anxiety \& Depression Scale (HADS) is 14-item measure of psychological distress. ${ }^{21}$ Items are rated on a 3 -point Likert scale and responses summed to yield 2 scores (anxiety and depression). The HADS is a common measure of psychological functioning in medical populations and demonstrates good reliability (Cronbach $\alpha$ from 0.65 to 0.90 , mean $=0.82$ ). A cutoff score of 8 is recommended for positive symptoms of each condition.

Gastroesophageal Reflux Disease Questionnaire The GERD Questionnaire (GERDQ) is a 6-item questionnaire that measures frequency of GERD symptoms, problems sleeping, and increased medication use during the previous week. ${ }^{22,23}$ Items are rated on a 4-point Likert scale (0 days to 4-7 days). Scores range from 0 to 18 with higher scores indicating more symptoms.

Following statistical analyses, the study team reviewed the BEDQ for potential item reduction and/or revision based on reliability statistics, ceiling and floor effects, and inter-item correlations. This study was reviewed and approved by the Institutional Review Board of Northwestern University.

\section{Statistical analyses}

Data were entered into SPSS version 23 (IBM-SPSS, Inc., Chicago IL, USA) for analysis. Descriptive statistics (percentages, mean + SD) evaluated the demographic and clinical variables of the sample by phase. Internal consistency and reliability of the BEDQ were evaluated using Cronbach's $\alpha$, inter-item correlation, and Guttman split-half reliability statistics. Tests for normal distribution (skewness, kertosis between -2.0 and +2.0 ) and mean + SD were conducted to identify potential ceiling (upper limit to values the scale can reliably specify) and floor (lower limit to values the scale can reliably specify) effects for each item of the BEDQ. ${ }^{24}$ Principle components factor analysis (PCFA) with varimax rotation evaluated the dimensionality of the BEDQ. Concurrent validity of the BEDQ was verified via Pearson's correlations with the VSI and HADS which measure variables consistently shown to be related to gastrointestinal symptoms (e.g. psychological distress) and the GERDQ. Construct validity was assessed via additional Pearson's correlations between the BEDQ, IRP, and DCI. Independent samples t-tests evaluated the construct validity of the BEDQ by measuring significant differences between patients with and without major motility disorders, mechanical obstructions, or presence of a large hiatal hernia.

The predictive ability of the BEDQ for major motor disorder, mechanical obstruction, or large hernia was evaluated two ways. First, three separate binary logistic regression analyses evaluated the predictive ability of the BEDQ for each diagnostic group. Variables were entered in a forward, conditional process. Nagelkerke R Square, Omnibus tests, and Hosmer and Lemeshow test evaluated the accuracy of the logistic regression model. Odds ratio with $95 \%$ confidence interval (CI) are reported. Second, three separate receiver operator characteristics (ROC) curve analyses established proposed cut-off scores for the BEDQ to identify each diagnostic group. A minimum acceptable area under the curve (AUC) was set for 0.70 . Cut points with sensitivity (true positive) and specificity (false positive) values are reported with the optimal cut point based on the Youden index, or the point on the ROC curve where sensitivity and specificity are maximized. ${ }^{25}$

\section{RESULTS}

A total of 1613 participants completed the study; 744 participants completed phase 1 and 869 phase 2. Study recruitment occurred between 2009 and 2012. Demographic and clinical characteristics of the sample are listed in Table 1. The sample was evenly divided between genders and heavily sampled from Caucasian and Non-Hispanic sources. On average, participants were middle-aged (range: 18-92 years) and overweight (BMI $\geq 25$, range: $15-44)$. Approximately $30 \%$ had major motor dysfunction, $20 \%$ mechanical obstruction, and $10 \%$ large hiatal hernia. No participants met the criteria for more than one category (e.g. mechanical obstruction and large hernia).

\section{Internal consistency, reliability, and factor structure of the BEDQ}

Overall, the BEDQ demonstrated excellent reliability $(\alpha>0.70)$ two of the three subscales (Table 2). The

Table 1 Demographic and clinical characteristics of study sample

\begin{tabular}{lcc}
\hline & $\%(N)$ or mean \pm SD \\
\cline { 2 - 3 } Variable & Part $1(n=744)$ & Part $2(n=869)$ \\
\hline Gender & & \\
$\quad$ Male & $43.0 \%(320)$ & $39.6 \%(344)$ \\
Female & $51.4 \%(383)$ & $59.8 \%(520)$ \\
Race & & \\
$\quad$ Caucasian & $87.9 \%(655)$ & $90.0 \%(782)$ \\
$\quad$ African/American & $8.1 \%(60)$ & $7.0 \%(61)$ \\
$\quad$ Other & $3.9 \%(30)$ & $3.0 \%(26)$ \\
Ethnicity & & \\
$\quad$ Hispanic & $5.4 \%(40)$ & $6.1 \%(53)$ \\
$\quad$ Non-Hispanic & $92.8 \%(691)$ & $93.9 \%(816)$ \\
Age & $48.73 \pm 14.9$ & $52.76 \pm 16.1$ \\
Body mass index & - & $27.49 \pm 6.0$ \\
Major motor dysfunction & - & $28.9 \%(251)$ \\
Mechanical obstruction & - & $18.3 \%(159)$ \\
Large hiatal hernia & - & $10.1 \%(88)$ \\
\hline
\end{tabular}


'impaction' scale demonstrated poor consistency $(\alpha=0.18)$. However, internal consistency was excellent for the total scale, with the impaction items included and removed (both >0.90). Correlations between BEDQ questions were within the acceptable cut-off range $(0.30-0.80)$ for all items. No significant ceiling or floor effects were noted for the individual scale items with the exception of the question measuring emergency room visits due to food impaction (92\% responded 'never'; floor effect).

Based on the poor consistency of the 'impaction' items, two separate PCFA evaluated the scale's dimensions. The first PCFA included all scale items and resulted in a single scale (Eigenvalue $=5.53,55 \%$ of the variance explained). Item correlations ranged from 0.24 (ER visits in past year) to 0.84 (Trouble swallowing liquids). The second PCFA removed the two impaction items, also resulting in a single scale (Eigenvalue $=5.17,65 \%$ of the variance explained). Item correlations ranged from 0.69 (Coughing or choking while swallowing foods) to 0.85 (Trouble swallowing liquids).

\section{Concurrent, construct, and predictive validity of the BEDQ}

The BEDQ demonstrated adequate concurrent validity as exhibited by significant correlations with outcomes measures included in the study (Table 3). The BEDQ was moderately correlated with related constructs measured by the HADS, VSI, and GERDQ, with the largest correlation with depression $(r=0.29, p<0.05)$. The relationship with BMI was non-significant, however, BMI did show a small correlation with the GERDQ $(r=0.18, p<0.05)$.

Construct validity is supported by patients with major motor dysfunction reporting significantly higher BEDQ scores than those without, $t(867)=-11.02$, $p<0.001$ (mean (SD): yes $=18.1 \quad(12.1), \quad$ no $=9.2$ (10.2)), those with mechanical obstruction scoring higher than those without, $t(458)=-3.46, p=0.001$ (yes $=12.6(10.8)$, no $=8.3(10.1))$, and those with a

Table 2 Internal consistency and split-half reliability statistics for the BEDQ

\begin{tabular}{lll}
\hline & Cronbach $\alpha$ & $\begin{array}{l}\text { Guttman split-half } \\
\text { coefficient }\end{array}$ \\
\hline Symptom frequency (items 1-5) & 0.872 & 0.808 \\
Symptom severity (items 8-10) & 0.861 & 0.755 \\
Impaction (items 6 and 7) & 0.180 & 0.180 \\
Total scale with items 6 and 7 & 0.901 & 0.865 \\
Total scale less items 6 and 7 & 0.917 & 0.898 \\
\hline
\end{tabular}

large hiatal hernia scoring higher than those without, $t$ $(453)=2.02, p=0.04$ (yes $=9.5(10.4)$, no $=7.1(9.5)$ ). Significant correlations exist between the BEDQ and adequacy of esophagogastric junction (EGJ) relaxation with swallowing (i.e. IRP value; $r=0.33, p<0.01$ ) and strength of distal esophageal contraction (i.e. DCI value; $r=0.11, p<0.05)$, further supporting the BEDQ's validity.

The predictive ability of the BEDQ for two of the three diagnostic categories was small but significant (Table 4). Patients with higher scores on the BEDQ are 1.1 times more likely to have major motor dysfunction and 1.04 times more likely to have mechanical obstruction when controlling for GERDQ, VSI, and HADS scores. No significant predictive relationship existed between the BEDQ and large hiatal hernia. Receiver operator characteristics curve analyses yielded a significant and acceptable AUC for major motor dysfunction (AUC $=0.73$; Fig. 1) but not for mechanical obstruction (AUC $=0.64$ ) nor large hiatal hernia (AUC $=0.35$ ). Table 5 outlines proposed cut scores for the BEDQ and diagnosing major motor dysfunction. The best cut point that maximizes (sensitivity + specificity) is a score of 10 on the BEDQ.

Table 3 Pearson's correlations of BEDQ with heartburn symptoms, visceral sensitivity, psychological variables, and BMI

\begin{tabular}{llc}
\hline Measure & $r$ & $p$ \\
\hline Visceral Sensitivity Index & 0.27 & $<0.001$ \\
HADS - anxiety & 0.22 & $<0.001$ \\
HADS - depression & 0.29 & $<0.001$ \\
Gastroesophageal Reflux Disease Questionnaire & 0.21 & $<0.001$ \\
Body mass index & 0.09 & 0.18 \\
\hline
\end{tabular}

Table 4 Logistic regression analyses of BEDQ for major motor dysfunction and mechanical obstruction

\begin{tabular}{|c|c|c|c|}
\hline & OR & $95 \% \mathrm{CI}$ & $p$ \\
\hline \multicolumn{4}{|l|}{ Major motor disorder } \\
\hline BEDQ & 1.06 & $1.04-1.09$ & $<0.001$ \\
\hline VSI & & & 0.09 \\
\hline GERDQ & & & 0.98 \\
\hline HADS - anxiety & & & 0.30 \\
\hline HADS - depression & & & 0.88 \\
\hline \multicolumn{4}{|l|}{ Mechanical obstruction } \\
\hline BEDQ & 1.04 & $1.01-1.07$ & 0.02 \\
\hline VSI & & & 0.55 \\
\hline GERDQ & & & 0.81 \\
\hline HADS - anxiety & & & 0.80 \\
\hline HADS - depression & & & 0.24 \\
\hline
\end{tabular}

VSI, GERDQ, and HADS were removed from each regression model due to non-significance. 


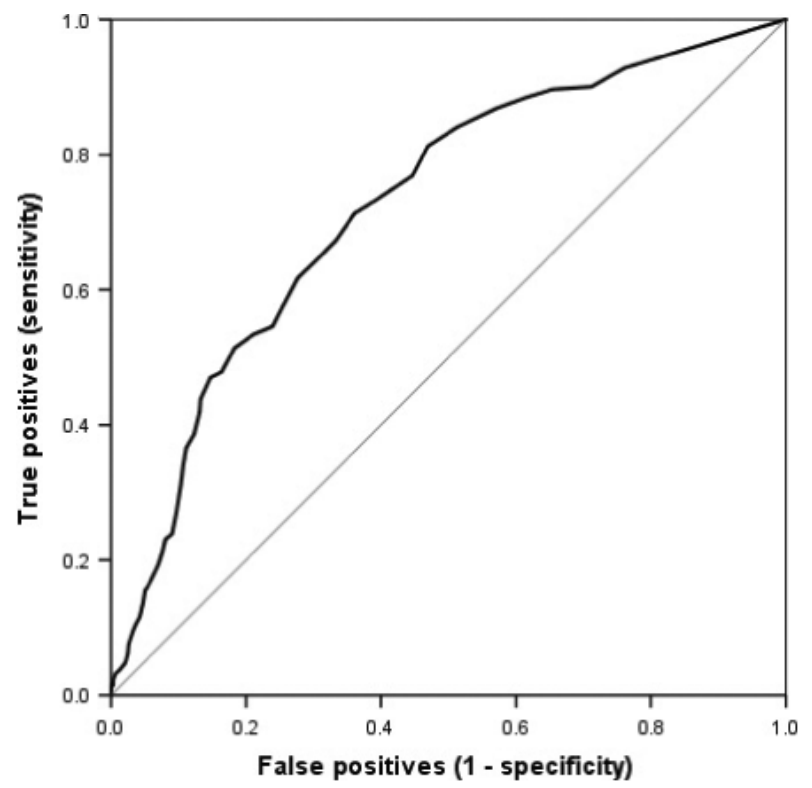

Figure 1 ROC curve for presence of a major motor disorder. AUC $=0.73,95 \%$ confidence interval $=0.69-0.76, p<0.001$.

Table 5 Cut points for BEDQ as predictor of major motor disorder

\begin{tabular}{lll}
\hline Cut point & Sensitivity & Specificity \\
\hline $4.5^{\star}$ & 0.90 & 0.43 \\
5.5 & 0.84 & 0.49 \\
6.5 & 0.81 & 0.53 \\
7.5 & 0.77 & 0.55 \\
8.5 & 0.73 & 0.61 \\
9.5 & 0.71 & 0.64 \\
10.5 & 0.67 & 0.67 \\
11.5 & 0.65 & 0.69 \\
\hline
\end{tabular}

${ }^{\star}$ First score to yield 0.90 or greater sensitivity.

\section{Final questionnaire design}

The final version of the BEDQ retains the original 10 items evaluating frequency and severity of dysphagia symptoms. Based on 2009 FDA guidelines for scale development, the timeframe for each scale was modified from its original 30 or 180 days to 14 days for frequency and severity, while 1 year was retained for the impaction questions due to the likely infrequent nature of these events in a 14 day period. An additional change for the frequency and severity scales includes qualifier responses to determine if respondents are able to eat each food type should a patient avoid a particular food category (e.g. solids) altogether. If a respondent is unable to eat a type of food, they are instructed to check the 'Cannot Eat' column, which automatically receive the maximum score (5 out of 5). Total scores range from 0 to 40 . Further validation of the BEDQ in its modified form is warranted to ensure its strong reliability and validity is retained.

\section{DISCUSSION}

We found the BEDQ to be a reliable and valid unidimensional measure of dysphagia severity in a large cohort of GERD and dysphagia patients visiting an outpatient gastroenterology practice. The measure allows for rapid assessment (under $2 \mathrm{~min}$ ) of selfreported symptom severity and frequency. Measures of internal consistency, split-half reliability, and construct validity were at or above established guidelines for scale development.

The BEDQ was modestly correlated with gastroesophageal reflux symptoms, visceral hypersensitivity, and psychological distress, all of which support construct validity. ${ }^{26-29}$ The BEDQ also identified differences in scores between patients with a major motor disorder, mechanical obstruction, or the presence of a large hernia. While the odds ratios are small, the BEDQ may significantly predict major motor dysfunction and mechanical obstruction in patients. Clinical utility of this predictive ability should be considered with caution as the corresponding effect size for an odds ratio less than 1.5 is likely very small. However, ROC curve analyses only maintain a reasonable, fair predictive ability of the BEDQ for major motor dysfunction with a suggested score of 10 to identify patients with a positive diagnosis. Additional studies are warranted to understand the diagnostic accuracy and reliability of the BEDQ to distinguish patients with major motor dysfunction from those who do not.

Internal consistency for the BEDQ was excellent, even with the inclusion of 2 scale items that demonstrated poor consistency and floor effects. Because the 'impaction' scale exhibited poor internal consistency but did not reduce the overall reliability of the BEDQ, the two items were converted to open-ended response to record the number of times each scenario had occurred in the past year (replacing the original Likert scale) and removed from the total score summation. Based on consensus from two gastroenterologists (JP, $\mathrm{PK})$, guidelines to interpret responses to the impaction items were developed as follows: in all patients, if at least $1 \mathrm{ER}$ visit for food impaction is present, more thorough evaluation is warranted. For patients who have yet to receive an esophageal diagnosis, one or more self-limited food impactions warrants endoscopic evaluation. Finally, in patients who have a known esophageal disorder such as EoE or achalasia, or an esophageal stricture, greater than two self-limited food impactions over 12 months would necessitate further 
evaluation and consideration of intervention. These two 'impaction' questions function to gauge esophageal dysphagia severity over the past year and bring additional clinical utility to the BEDQ to guide diagnostic intervention. See Appendix S1 for the final version of the BEDQ.

While existing assessment tools, such as the MDQ30, provide in-depth information about esophageal dysphagia symptoms that is critical for clinical research, their implementation in day-to-day clinical practice can be challenging. Like the GERDQ, ${ }^{22}$ the BEDQ allows for evaluation in the clinical setting in under $5 \mathrm{~min}$. The short length of the BEDQ also reduces the likelihood of issues common to assessment scales such as fatigue, straight-line answering, or incomplete data. ${ }^{30,31}$ However, some research suggests that shorter questionnaires are not necessarily superior to longer assessment tools. ${ }^{32}$

There are some limitations to the present study that should be taken into consideration. The study sample was primarily Caucasian and non-Hispanic. While data regarding racial and ethnic differences for esophageal dysphagia are limited, some studies suggest racial differences exist in dysphagia prevalence ${ }^{33,34}$ and symptom presentation. ${ }^{35}$ Therefore, caution should be applied when using the BEDQ among racial and ethnic minorities until it is evaluated in more diverse populations. The diagnosis of GERD may not typically present with dysphagia symptoms, and the dysphagia NOS group is poorly defined in terms of underlying esophageal diagnosis (e.g. achalasia, eosinophilic esophagitis) so the relationship between the BEDQ and the underlying esophageal disorder is uncertain. Only cross-sectional data for the BEDQ were collected so the temporal stability of BEDQ scores is not known. Additional validation of the BEDQ is warranted. This includes evaluating the accuracy of suggested diagnostic cut-off scores and impaction guidelines with manometric and other pathophysiological data, comparison of the BEDQ to the MDQ30, and test-retest evaluation.
In summary, we have developed a rapid tool to assess frequency and severity of dysphagia symptoms and food impactions in the outpatient setting. This tool could be utilized to follow patients before and after intervention and help determine which patients will require further evaluation and treatment; prospective trials are needed to confirm this use. We are optimistic that this tool could be utilized in large population based studies as it can easily be recorded in the EMR while other, more comprehensive measures such as the MDQ are utilized in settings requiring more detailed assessment. Future studies will determine whether improvement or normalization of BEDQ score is associated with good treatment outcomes in both structural and non-structural esophageal dysphagia.

\section{ACKNOWLEDGMENTS}

The authors want to thank the patients who participated in this study, the laboratory technicians who assisted with data collection, and physician colleagues who identified potential participants for recruitment.

\section{FUNDING}

Peter J Kahrilas and John E Pandolfino were supported by R01 DK079902 (JEP) and R01DK56033 (PJK) from the US National Institutes of Health (NIH).

\section{CONFLICTS OF INTEREST}

The authors have no competing interests to report.

\section{AUTHOR CONTRIBUTION}

\begin{abstract}
TT statistical analyses, data interpretation, manuscript preparation; MR data interpretation, manuscript preparation; JS data collection and interpretation, manuscript preparation; PK study design, BEDQ development, data interpretation, manuscript preparation; LK BEDQ development, data interpretation, manuscript preparation; BD BEDQ development, manuscript preparation; JP study design, BEDQ development, data interpretation, manuscript preparation.
\end{abstract}

\section{REFERENCES}

1 ASGE Standards of Practice Committee, Pasha SF, Acosta RD, Chandrasekhara V, Chathadi KV, Decker GA, Early DS, Evans JA, Fanelli RD et al. The role of endoscopy in the evaluation and management of dysphagia. Gastrointest Endosc 2014; 79: 191-201.

2 CullingfordGL, Carr-LockeDL. Should endoscopy rather than radiology be the primary investigation for dysphagia? Med I Aust 1989; 151: 238.

3 Longstreth GF, Longstreth KJ, Yao JF. Esophageal food impaction: epidemiology and therapy. A retrospective, observational study. Gastrointest Endosc 2001; 53: 193-8.

4 Benjamin SB. Esophageal foreign bodies and food impactions. Gastroenterol Hepatol 2008; 4: 546-8.

5 Desai TK, Stecevic V, Chang $\mathrm{CH}$, Goldstein NS, Badizadegan $\mathrm{K}$,
Furuta GT. Association of eosinophilic inflammation with esophageal food impaction in adults. Gastrointest Endosc 2005; 61: 795-801.

6 Kahrilas PJ, Smout AJ. Esophageal disorders. Am J Gastroenterol 2010; 105: 747-56.

7 Kerlin P, Jones D, Remedios M, Campbell C. Prevalence of eosinophilic esophagitis in adults with food bolus obstruction of the esophagus. I Clin Gastroenterol 2007; 41: 356-61. 
8 Sperry SL, Crockett SD, Miller CB, Shaheen NJ, Dellon ES. Esophageal foreign-body impactions: epidemiology, time trends, and the impact of the increasing prevalence of eosinophilic esophagitis. Gastrointest Endosc 2011; 74: 985-91.

9 Bredenoord AJ, Fox M, Kahrilas PJ, Pandolfino JE, Schwizer W, Smout AJ; International High Resolution Manometry Working Group. Chicago classification criteria of esophageal motility disorders defined in high resolution esophageal pressure topography. Neurogastroenterol Motil 2012; 24 (Suppl. 1): 57-65.

10 Burgess NG, Wyeth JW. An audit of combined multichannel intraluminal impedance manometry in the assessment of dysphagia. I Gastroenterol Hepatol 2011; 26 (Suppl. 3): 79-82.

11 Feussner H, Kauer W, Siewert JR. The place of esophageal manometry in the diagnosis of dysphagia. Dysphagia 1993; 8: 98-104.

12 Galmiche JP, Clouse RE, Balint A, Cook IJ, Kahrilas PJ, Paterson WG, Smout AJ. Functional esophageal disorders. Gastroenterology 2006; 130: 1459-65.

13 Kumar AR, Katz PO. Functional esophageal disorders: a review of diagnosis and management. Expert Rev Gastroenterol Hepatol 2013; 7: 453-61.

14 Speyer R, Cordier R, Kertscher B, Heijnen BJ. Psychometric properties of questionnaires on functional health status in oropharyngeal dysphagia: a systematic literature review. Biomed Res Int 2014; 2014: 458678.

15 Grudell AB, Alexander JA, Enders FB, Pacifico R, Fredericksen M, Wise JL, Locke GR 3rd, Arora A et al. Validation of the Mayo Dysphagia Questionnaire. Dis Esophagus 2007; 20: 202-5.

16 Kwiatek MA, Kiebles JL, Taft TH, Pandolfino JE, Bove MJ, Kahrilas PJ, Keefer L. Esophageal symptoms questionnaire for the assessment of dysphagia, globus, and reflux symptoms: initial development and validation. Dis Esophagus 2011; 24: 550-9.

17 Dellon ES, Irani AM, Hill MR, Hirano I. Development and field testing of a novel patient-reported outcome measure of dysphagia in patients with eosinophilic esophagitis. Aliment Pharmacol Ther 2013; 38: 634-42.

18 Schoepfer AM, Straumann A, Panczak R, Coslovsky M, Kuehni CE, Maurer E, Haas NA, Romero Y et al. Development and validation of a symptom-based activity index for adults with eosinophilic esophagitis. Gastroenterology 2014; 147: 1255-66 e21.

19 Straumann A, Conus S, Degen L, Felder S, Kummer M, Engel H, Bussmann C, Beglinger C. Budesonide is effective in adolescent and adult patients with active eosinophilic esophagitis. Gastroenterology 2010; 139: $1526-37,37$ e1.

20 Labus JS, Bolus R, Chang L, Wiklund I, Naesdal J, Mayer EA, Naliboff BD. The Visceral Sensitivity Index: development and validation of a gastrointestinal symptom-specific anxiety scale. Aliment Pharmacol Ther 2004; 20: 89-97.

21 Zigmond AS, Snaith RP. The hospital anxiety and depression scale. Acta Psychiatr Scand 1983; 67: 361-70.

22 Jones R, Junghard O, Dent J, Vakil N, Halling $\mathrm{K}$, Wernersson $\mathrm{B}$, Lind $\mathrm{T}$. Development of the GerdQ, a tool for the diagnosis and management of gastro-oesophageal reflux disease in primary care. Aliment Pharmacol Ther 2009; 30: 1030-8.

23 Bai Y, Du Y, Zou D, Jin Z, Zhan X, Li ZS, Yang Y, Liu Y et al. Gastroesophageal Reflux Disease Questionnaire (GerdQ) in real-world practice: a national multicenter survey on 8065 patients.

I Gastroenterol Hepatol 2013; 28: 626-31.

24 Winters JJ Jr. Proposed analyses of floor and ceiling effects. Am I Ment Defic 1972; 77: 296-300.

25 Kumar R, Indrayan A. Receiver operating characteristic (ROC) curve for medical researchers. Indian Pediatr 2011; 48: 277-87.

26 Sharma A, Van Oudenhove L, Paine P, Gregory L, Aziz Q. Anxiety increases acid-induced esophageal hyperalgesia. Psychosom Med 2010; 72: 802-9.

27 Szczesniak MM, Fuentealba SE, Cook IJ. Acid sensitization of esophageal mucosal afferents: implication for symptom perception in patients across the gastroesophageal reflux disease spectrum. Clin I Pain 2013; 29: 70-7.

28 van Tilburg MA, Palsson OS, Whitehead WE. Which psychological factors exacerbate irritable bowel syndrome? Development of a comprehensive model. I Psychosom Res 2013; 74: 486-92.

29 Verdonschot RJ, Baijens LW, Serroyen JL, Leue C, Kremer B. Symptoms of anxiety and depression assessed with the hospital anxiety and depression scale in patients with oropharyngeal dysphagia. I Psychosom Res 2013; 75 : 451-5.

30 Rolstad S, Adler J, Ryden A. Response burden and questionnaire length: is shorter better? A review and metaanalysis. Value Health $2011 ; \mathbf{1 4}$ : 1101-8.

31 Edwards P, Roberts I, Sandercock P, Frost C. Follow-up by mail in clinical trials: does questionnaire length matter? Control Clin Trials 2004; 25:31-52.

32 Subar AF, Ziegler RG, Thompson FE, Johnson CC, Weissfeld JL, Reding D, Kavounis KH, Hayes RB; Prostate, Lung, Colorectal, and Ovarian Cancer Screening Trial Investigators. Is shorter always better? Relative importance of questionnaire length and cognitive ease on response rates and data quality for two dietary questionnaires. Am J Epidemiol 2001; 153: 404-9.

33 Wilkins T, Gillies RA, Thomas AM, Wagner PJ. The prevalence of dysphagia in primary care patients: a HamesNet Research Network study. I Am Board Fam Med 2007; 20: 144-50.

34 Bussell SA, Gonzalez-Fernandez M. Racial disparities in the development of dysphagia after stroke: further evidence from the Medicare database. Arch Phys Med Rehabil 2011; 92: 737-42.

35 Bohm M, Malik Z, Sebastiano C, Thomas R, Gaughan J, Kelsen S, Richter JE. Mucosal eosinophilia: prevalence and racial/ethnic differences in symptoms and endoscopic findings in adults over 10 years in an urban hospital. I Clin Gastroenterol 2012; 46: 567-74.

\section{SUPPORTING INFORMATION}

Additional Supporting Information may be found online in the supporting information tab for this article:

Appendix S1 Final version of the brief esophageal dysphagia questionnaire. 